\title{
REAL-TIME IMAGE SUBTRACTION USING A DOUBLE-PHASE CONJUGATE MIRROR
}

BY N. L. SWANSON

M. E. STEFANOV

SYSTEMS RESEARCH AND TECHNOLOGY DEPARTMENT

NOVEMBER 1998

Approved for public release, distribution is unlimited.

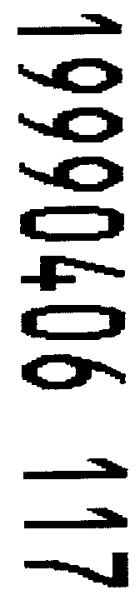


Public reporting burden for this collection of information is estimated to average 1 hour per response, including the time for reviewing instructions, search existing data sources, gathering and maintaining the data needed, and completing and reviewing the collection of information. Send comments regarding this burden or any other aspect of this collection of information, including suggestions for reducing this burden, to Washington Headquarters Services. Directorate for information Operations and Reports, 1215 Jefferson Davis Highway, Suite 1204, Arlington, VA 222024302, and to the Office of Management and Budget, Paperwork Reduction Project (0704-0188), Washington, DC 20503.
1. AGENCY USE ONLY (Leave blank)
2. REPORT DATE
3. REPORT TYPE AND DATES COVERED

November 1998

Final

\section{TITLE AND SUBTITLE}

5. FUNDING NUMBERS

Real-Time Image Subtraction Using a Double-Phase Conjugate Mirror

6. AUTHOR(s)

N. L. Swanson, M. E. Stefanov

7. PERFORMING ORGANIZATION NAME(S) AND ADDRESS(ES)

Commander

Naval Surface Warfare Center

Dahlgren Division (Code B20)

17320 Dahlgren Road

Dahlgren, VA 22448-5100
Commander

NSWCDD (Code R23)

Coastal Systems Station

6703 West Highway 98

Panama City, FL 32407-7001
8. PERFORMING ORGANIZATION REPORT NUMBER

NSWCDD/TR-98/136
9. SPONSORINGMONITORING AGENCY NAME(S) AND ADDRESS(ES)

Office of Naval Research

800 N. Quincy Street

Arlington, VA

\section{SUPPLEMENTARY NOTES}

\section{2a. DISTRIBUTION/AVAILABILITY STATEMENT}

Approved for public release; distribution is unlimited. 12b. DISTRIBUTION CODE

\section{ABSTRACT (Maximum 200 words)}

A method has been demonstrated by holographic interference in photo-refractive media for real-time image subtraction requiring only a single pass through an aberrating medium. Image enhancement by passing the conjugate beam back through the aberrator (double pass) has been previously demonstrated. For various applications, a single pass is required. The theory and experiments for double- and single-pass arrangements are presented.

\section{SUBJECT TERMS}

15. NUMBER OF PAGES

optical-phase conjugation, double-phase conjugation, image subtraction, image enhancement

17. SECURITY CLASSIFICATION
OF REPORTS
UNCLASSIFIED

18. SECURITY CLASSIFICATION
OF THIS PAGE
UNCLASSIFIED

19. SECURITY CLASSIFICATION OF ABSTRACT

UNCLASSIFIED

\section{2}

16. PRICE CODE

20. LIMITATION OF ABSTRACT

UL 


\section{FOREWORD}

A method has been demonstrated by holographic interference in photo-refractive media for real-time image subtraction requiring only a single pass through an aberrating medium. Image enhancement by passing the conjugate beam back through the aberrator (double pass) has been previously demonstrated. For various applications, a single pass is required. The theory and experiments for double- and single-pass arrangements are presented.

This project was funded by the Office of Naval Research (ONR) and was completed in September 1991 at Naval Surface Warfare Center, Dahlgren Division (NSWCDD), Coastal Systems Station, Panama City, Florida. The ONR project number was R00N0. The Coastal Systems Station project number was 40001 . The funding document identification number was N0001491WX4E004.

The authors would like to express their appreciation to E. J. Sharp and his research group at the Night Vision Laboratory, Fort Belvoir, Virginia, and to R. R. Neurgaonkar and his research group at Rockwell International Laboratories, Thousand Oaks, California, for their assistance in conducting this project.

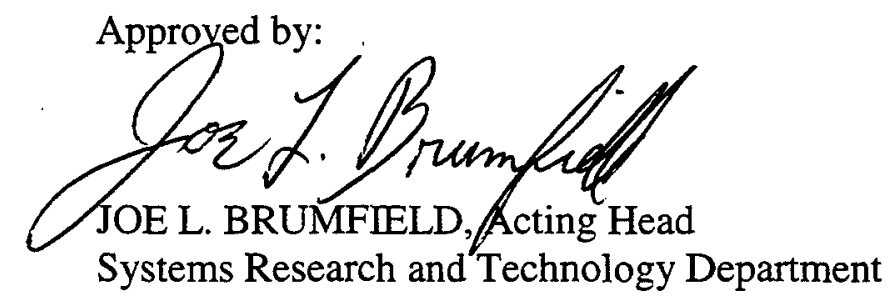




\section{CONTENTS}

$\underline{\text { Page }}$

INTRODUCTION ...................................................................................................

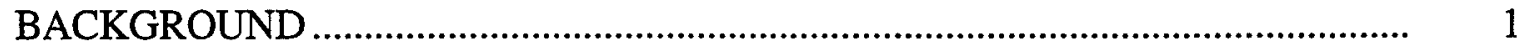

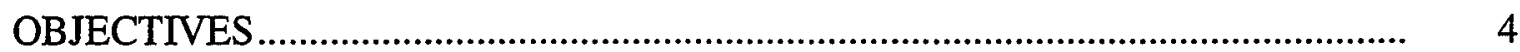

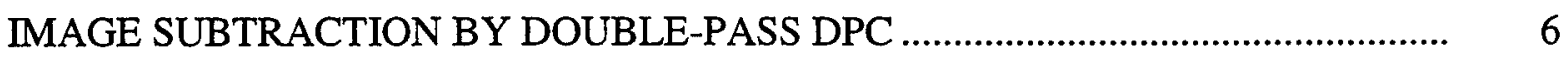

IMAGE SUBTRACTION BY SINGLE-PASS DPC ....................................................... 8

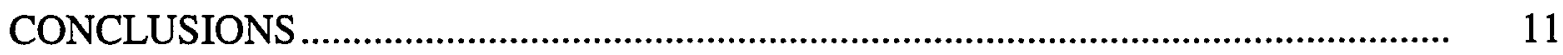

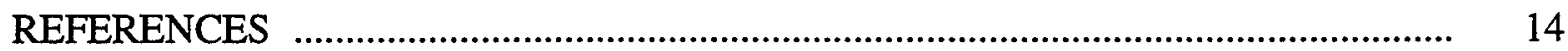

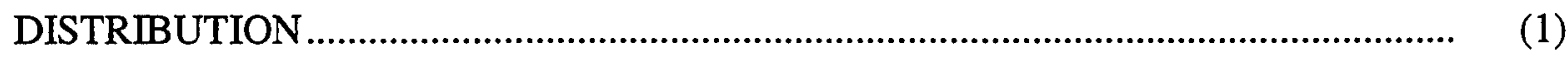

\section{ILLUSTRATIONS}

Figure $\quad \underline{\text { Page }}$

1 STANDARD MIRROR VS. PHASE CONJUGATE MIRROR ……............. 1

2 REMOVAL OF ABERRATION BY OPTICAL-PHASE

CONJUGATION ................................................................................... 5

3 EXPERIMENTAL SETUP, DOUBLE PASS ................................................... 6

$4 \quad$ EXPERIMENTAL SETUP, SINGLE PASS ................................................ 9

$5 \quad$ SUBTRACTION OF TWO GAUSSIAN BEAMS ……................................. 12 


\section{INTRODUCTION}

\section{BACKGROUND}

Optical-phase conjugation is a process by which each plane wave in an arbitrary beam of light precisely reverses both its direction and overall phase factor. This process is mediated through nonlinear optical interactions in certain materials. The phase difference between any two points of the conjugate beam has a sign opposite to that of the phase difference between the same points of the original beam. Conjugation is the mathematical operation of changing a phase sign. This is why the operation is referred to as optical-phase conjugation. In a typical Fresnel mirror, the reflection obeys several well-known relationships; i.e., the angle of reflection equals the angle of incidence and a diverging wavefront will continue to diverge upon reflection. For a phase conjugate mirror, the reflection will retrace its path and the once divergent beam will converge with the same angular relationships as the original beam (Figure 1).

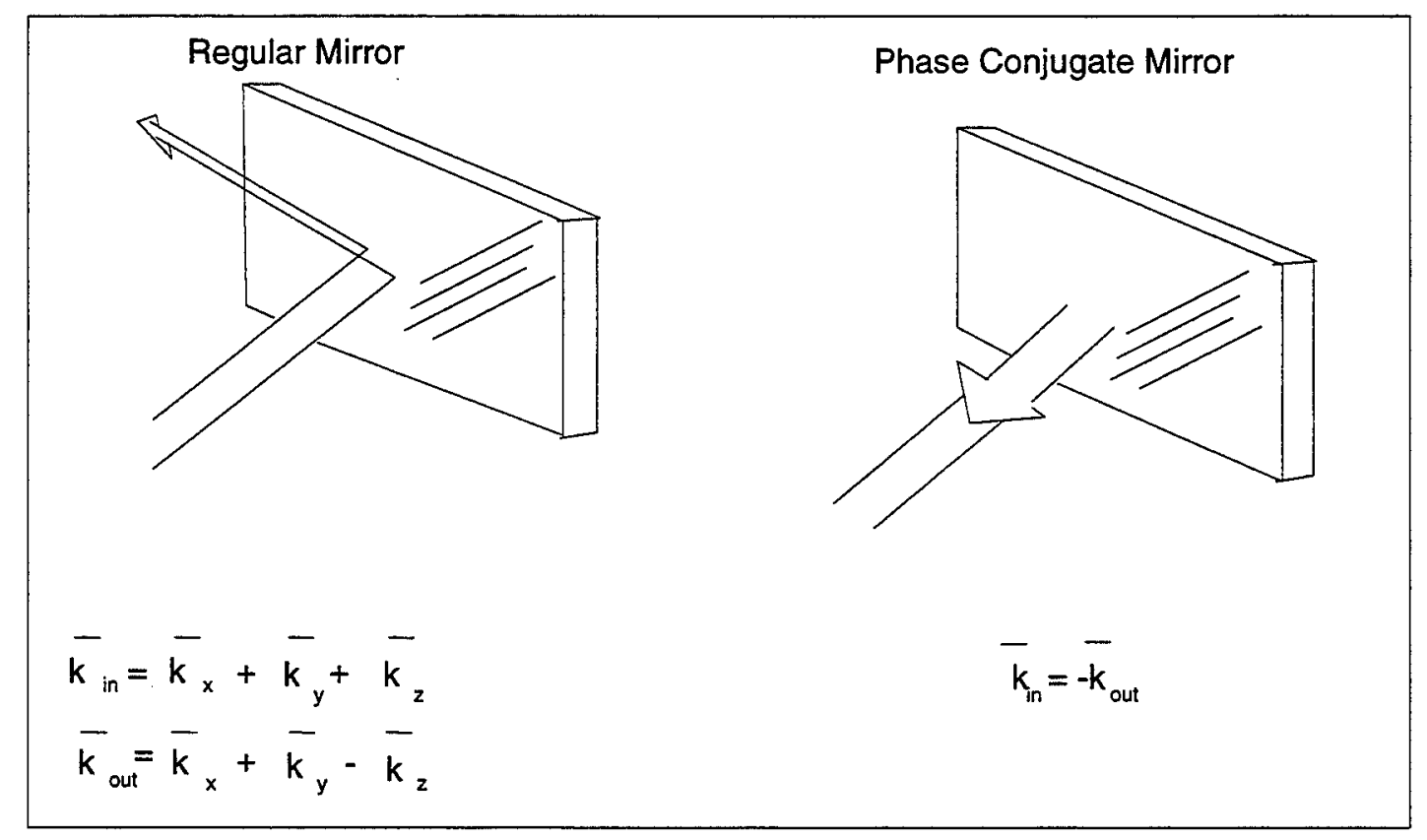

FIGURE 1. STANDARD MIRROR VS. PHASE CONJUGATE MIRROR 
Mathematically the distortion removing properties of a phase conjugate beam are well understood. ${ }^{1}$ Assume a monochromatic beam propagating through a composite medium whose dielectric permittivity is defined by $\varepsilon(\mathbf{r})$. The scalar $(+z)$ propagating beam is

$$
\overrightarrow{\mathbf{E}}_{1}(\overrightarrow{\mathbf{r}}, t)=\frac{1}{2} \xi_{1}(\overrightarrow{\mathbf{r}}) e^{-i(w t-k z)}+\text { c.c. }
$$

In the limit of slow spatial and temporal variations, the scalar wave equation becomes

$$
\nabla^{2} \xi_{1}+\left(\omega^{2} \mu \varepsilon(\mathbf{r})-k^{2}\right) \xi_{1}+2 i k \frac{\left(\partial \xi_{1}^{*}\right)}{(\partial z)}=0
$$

The complex conjugate of Equation (2) can be written as

$$
\nabla^{2} \xi_{1}^{*}+\left(\omega^{2} \mu \varepsilon(\mathbf{r})-\mathrm{k}^{2}\right) \xi_{1}^{*}-2 i \mathrm{k} \frac{\left(\partial \xi_{1}\right)}{(\partial z)}=0
$$

Equation (3) is the wave equation for a wave propagating in the $-z$ direction described by

$$
\vec{E}_{2}(\overrightarrow{\mathbf{r}}, t)=\frac{1}{2} \xi_{2} e^{-i(\omega t+k z)}+c . c .
$$

with the constraint

$$
\xi_{2}(\mathbf{r})=a \xi_{1}^{*}(\mathbf{r})
$$

where $a$ is an arbitrary constant. Therefore a wave travelling in the opposite direction $\xi_{2}$ with a complex amplitude, which is the complex conjugate of $\xi_{1}$ (within an arbitrary multiplicative constant), is also a solution of the wave equation obeyed by $\xi_{1}$. Physically this implies that a phase conjugate mirror can generate a beam $E_{2}$, which is the phase conjugate of an arbitrarily distorted beam $E_{1}$ (within an arbitrary multiplicative constant). $E_{2}$ will propagate opposite to $E_{1}$ and its amplitude $\xi_{2}$ will be the complex conjugate of $\xi_{1}$. It is apparent that the wavefronts of $E_{2}$ coincide everywhere with those of $E_{1}$. It should be noted that $E_{1}$ and its reflected conjugate $E_{2}$ have the same time dependence $\exp (-i w t)$ and it is only the spatial part $\xi_{1}(\mathbf{r}) \exp (i k z)$ that is reversed. These equations hold as long as $\varepsilon(\mathbf{r})$ is real. If the propagation material is lossy or amplifying, the equations are not true unless the loss or gain is independent of $\mathbf{r}$. For this purpose, the assumption that the propagation material is lossless and non-amplifying is valid.

The photorefractive effect is the mechanism for phase conjugate processes. It is caused by impurities, vacancies, or defects in the crystals that act as charge donors and acceptors. When light strikes the crystal, these extra charges rearrange themselves. The present thinking favors two possible transport mechanisms. The first is that the charges, either electrons and/or holes, are excited into the closest energy band, become mobile, and are eventually retrapped within the crystal. The second mechanism is that of light-assisted tunneling or band hopping between 
trapping sites. Regardless of the mechanism, if the photorefractive crystal is continuously irradiated with light within a proper bandwidth, the charges eventually arrange themselves in an equilibrium pattern. Since the charges have been trapped, they remain in this equilibrium pattern even after the light has been removed for extended periods. An interesting difference between photorefractive materials and other nonlinear optical materials is that their optical nonlinearity is independent of the intensity of the optical beams, thus achieving nonlinear effects even with relatively weak laser beams. The optical intensity determines the speed of the nonlinear response, not its magnitude.

When a volume phase grating is written in a photorefractive crystal, optical-phase conjugation follows. How a volume phase grating is established within the photorefractor is described as follows: Allow two coherent light beams to irradiate a crystal. The loosely bound charges become mobile and accumulate in the dark areas of the interference grating. Thus a nonuniform charge density $\rho(x)$ is formed. This charge density produces a static, spatially varying periodic electric field $\overrightarrow{\mathbf{E}}(x)$ within the crystal. Poisson's equation

$$
\vec{\nabla} \cdot \overrightarrow{\mathbf{E}}(x)=\left(\frac{4 \pi}{\varepsilon}\right) \rho(x)
$$

shows that, while the charge densities may be low, the electric fields in the crystal are on the order of $1000 \mathrm{v} / \mathrm{cm}$. This large localized field distorts the crystal lattice, which in turn alters the crystal's index of refraction $n(x)$. The change in the index of refraction is

$$
\Delta n(x)=-\left(\frac{n^{3} p}{2}\right) \overrightarrow{\mathrm{E}}(x)
$$

where $p$ is the Pockel's coefficient of the crystal. The Pockel's coefficients are elements of the crystal's third rank tensor $\mathbf{r}_{i j k}$, which is the reason why certain photorefractive materials exhibit such highly nonlinear effects. Therefore it is easily seen that if the light irradiating the crystal is spatially periodic, the variations in the refractive index $\Delta n(x)$ will have the same periodicity. The end result is that the crystal has now become a volume phase grating. Again it is this volume phase grating that produces the phase conjugate of the object beams.

This research exploited the double-phase conjugation (DPC) mechanisms in the photorefractive crystal strontium barium titanate. The double self-pumped phase conjugator, using barium titanate, was first demonstrated by Weiss, et al. ${ }^{2}$ at the Technion in Israel and independently at the University of Essex by Smout and Eason. ${ }^{3}$ In their initial work, both groups crossed two laser beams in the photorefractor and observed that there were two reflected beams, and they were the conjugates of the initial input beams. They also found two completely unexpected relationships. The first was that the reflected beams were conjugates of themselves and not each other. The second was that the effect worked best when the laser beams were the same wavelength but were incoherent with each other. This is in contrast with four-wave mixing phase conjugation. The DPC process happens by indirect means. The two beams cannot simply write a volume hologram together since they are mutually incoherent. The process occurs 
because each beam writes a hologram with its own scattered light. Once the holograms have been formed, the relevance of the coherence disappears. (To become more familiar with phase conjugation and photorefractive materials, see the introductory papers of References 4-6 and Chapter 11 of Reference 1.)

\section{OBJECTIVES}

This research effort investigated the feasibility of using phase conjugation phenomena for image enhancement. It has been shown ${ }^{7}$ that phase conjugation techniques can be used to correct for aberrations in images. The aberrator acts to change the phase of the wave at each point so that the transmission of a beam through an aberrator is

$$
T_{A}(\vec{r})=e^{i \phi(\vec{r})},
$$

where $\overrightarrow{\mathrm{r}}$ is the distance from the center of the beam in the plane perpendicular to the direction of propagation. Equation (8) is valid only for a phase-distorting aberrator. It is not valid if the aberrator induces a spatially varying amplitude structure in the beam. Figure 2 shows a schematic of this effect. The field incident on the aberrator is $E_{o}$. After passing through the aberrator, the attenuated field is $E_{o} T_{A}$. This field is reflected in the crystal grating, and the conjugated field is $E_{o}^{*} T_{A}^{*}$. Upon being transmitted back through the aberrator, the conjugated

field becomes $E_{o}^{*}\left|T_{A}\right|^{2}$. By Equation (8), $\left|T_{A}\right|^{2}=1$, and the aberration is removed. The conjugated field is the inverse of the field incident on the crystal so that the transmission back through the aberrator reverses the initial aberration. This technique requires a double pass through the aberrator.

For Navy applications, the beam is required to pass through the aberrator only once. The ability to resolve images distorted by propagation through a water medium requires the removal or undoing of the aberrations produced in the water. There are of course many sophisticated digital image processing techniques available for image enhancement. All of these methods have two things in common - they operate on the image after it has been recorded, and they process information in a serial fashion. The technical problem addressed is whether or not image enhancement through the removal of aberrations before the object is imaged and captured is possible (i.e., real-time image enhancement). The task was to determine whether or not it is possible to use this method in a single pass configuration for reducing the noise (backscatter, etc.) from underwater images in mine countermeasures. 


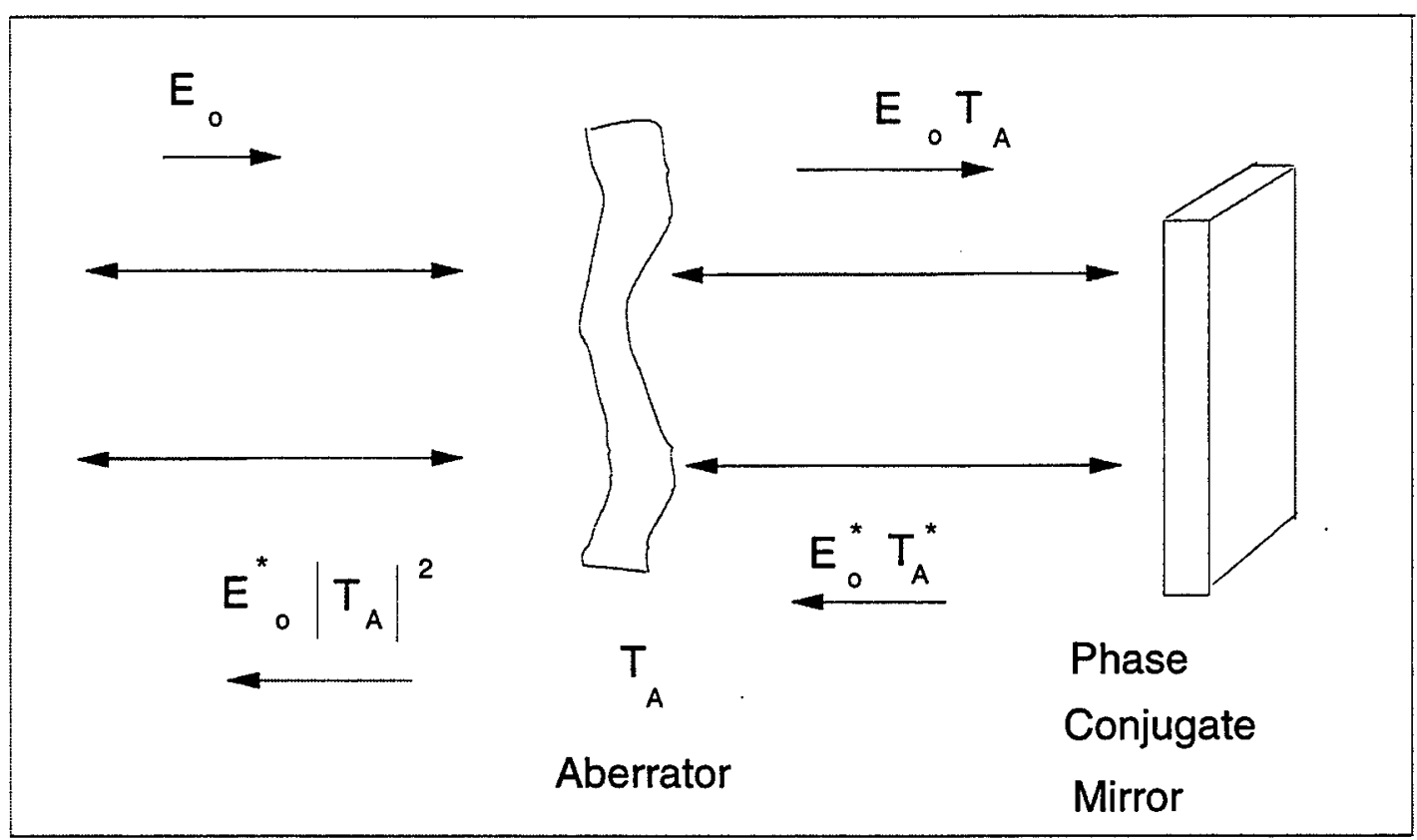

FIGURE 2. REMOVAL OF ABERRATION BY OPTICAL-PHASE CONJUGATION

The Navy and Marine Corps have an obvious need for locating mines and minefields. An airborne active imaging system has the potential for high payoff. If real-time image processing proves feasible, then the operational problem that must be addressed is whether or not its advantages can be realized in an operational system meeting the systems requirements. The operational requirements for airborne systems dictate large area and/or volume coverage and high sensitivity for an active optical system. This severely limits operational design and configuration. The impact of these constraints will be addressed in the conclusion of this report.

Although DPC techniques were used, it should be noted that there are many different physical mechanisms (three- or four-wave mixing, stimulated scattering, etc.) that permit one to implement the optical-phase conjugation process. Also, there are many types of materials for implementing these physical mechanisms. This work draws heavily on relatively few ${ }^{7-15}$ of the large number of publications available on both theory and experimental results. All of the studied processes that have been implemented for correction of static and dynamic aberrations in the atmospheric environment have a direct correlation to a water environment since the water can be considered an aberrator. 


\section{IMAGE SUBTRACTION BY DOUBLE-PASS DPC}

Image subtraction requiring a double pass through transparencies has been demonstrated in self-pumped phase conjugate mirrors ${ }^{13}$ and in DPC mirrors. ${ }^{14}$ In the DPC method, wherein the two pump beams also serve as the reference beams, alignment difficulties are greatly alleviated.

Figure 3 is a diagram of the experimental setup for image subtraction through recombination of the conjugate beams. The laser light passes through a polarizer and is expanded with a collimator. The beam is split at BS2 into two beams with fields $E_{\mathrm{a}}$ and $E_{\mathrm{b}} . E_{\mathrm{a}}$ passes through the target transparency $\mathrm{T} 1$ and $E_{\mathrm{b}}$ passes through target T2. Both are focussed into the crystal by lenses $\mathrm{L} 1$ and $\mathrm{L} 2$. The conjugate beams are reflected back in the same direction as the input beams and are recombined at BS2. BS2 has reflection and transmission coefficients $r$ and $t$ for light incident from one side of the cube and $r^{\prime}$ and $t^{\prime}$ for light incident from the other.

The fields $E_{1}$ and $E_{2}$ at the outputs of BS1 and BS2 are calculated as follows: The fields writing the grating, $E_{\mathrm{a}}$ and $E_{\mathrm{b}}$, are

$$
\begin{gathered}
E_{a}=\operatorname{tr} T_{1} E_{\text {in }} \\
E_{b}=t t T_{2} E_{\text {in }} \\
E_{a}^{*}=\text { conj of } E_{a} \\
E_{b}^{*}=\text { conj of } E_{b} .
\end{gathered}
$$

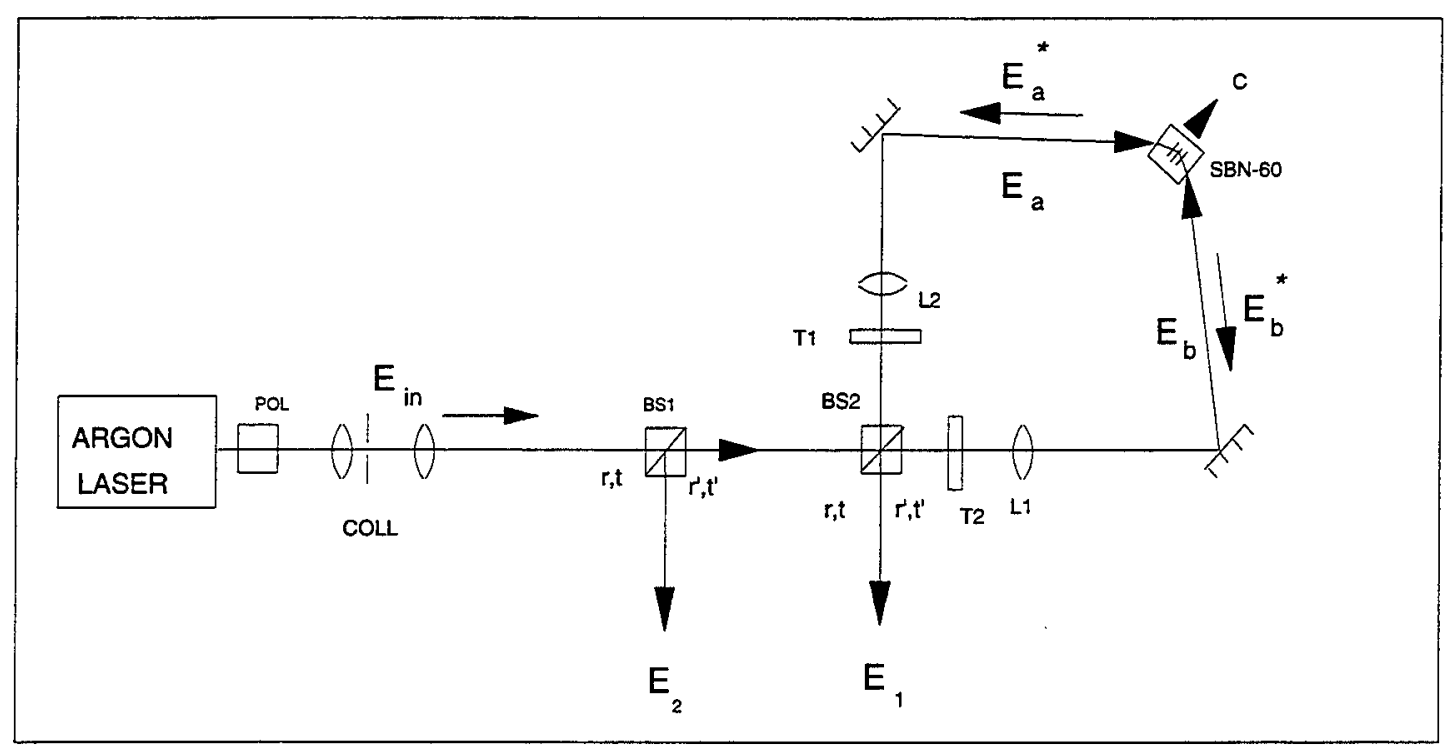

FIGURE 3. EXPERIMENTAL SETUP, DOUBLE PASS 
The field $E_{1}$ at the output of BS2 is the combined conjugates of $E_{\mathrm{a}}$ and $E_{\mathrm{b}}$ given by

$$
\begin{gathered}
E_{1}=\left(E_{a}^{*} t R_{1} T_{1}+E_{b}^{*} r^{\prime} R_{2} T_{2}\right) \\
=t^{*} E_{\mathrm{in}}^{*}\left(t r^{*}\left|T_{1}\right|^{2} R_{1}+t^{*} r^{\prime}\left|T_{2}\right|^{2} R_{2}\right),
\end{gathered}
$$

where $R_{1}$ and $R_{2}$ are the phase conjugate reflectivities of the conjugate mirror. From Stoke's principle of the time reversibility of light

$$
t^{*} r^{\prime}+r^{*} t=0
$$

where $t=t^{\prime}$. Applying Equation (11) to Equation (10) and assuming $\mathrm{R}_{1}=\mathrm{R}_{2}$ gives

$$
E_{1}=|t|^{2} E_{\text {in }}^{*} R^{*}\left(\left|T_{1}\right|^{2}-\left|T_{2}\right|^{2}\right)
$$

for the field. The detected intensity is the square amplitude of the field in Equation (12). The negative sign in Equation (12) indicates a phase difference of $\pi$ in the two conjugate beams. This setup can therefore be used to image the intensity difference between the two transparencies. This device is not sensitive to phase differences or to an optical path difference in the interferometer.

If there is only one target in one leg of the interferometer, Equation (12) becomes

$$
E_{1}=|t|^{2} E_{\text {in }}^{*} R^{*}\left(1-\left|T_{2}\right|^{2}\right),
$$

and image inversion takes place. If both target transparencies are removed, the beams are identical Gaussian and

$$
E_{1}=|t|^{2} E_{\text {in }}^{*} R r^{*}(1-1)=0 .
$$

The phase difference of $\pi$ in the conjugate beams causes complete destructive interference. This was observed in the laboratory.

The field $E_{2}$ at the output of BS1 is

$$
E_{2}=\left(E_{a}^{*} r r^{\prime} R_{1} T_{1}+E_{b}^{*} t^{\prime} r^{\prime} R_{2} T_{2}\right) .
$$

Inserting the values of $E_{a}^{*}$ and $E_{b}^{*}$ from Equation (9) gives

$$
E_{2}=r^{\prime} t^{*} E_{\text {in }}^{*}\left(|r|^{2}\left|T_{1}\right|^{2} R_{1}+|t|^{2}\left|T_{2}\right|^{2} R_{2}\right) .
$$


By applying conservation of energy, $|r|^{2}+|t|^{2}=1$, to Equation (16) and again assuming $R_{1}=R_{2}$ then

$$
E_{2}=r^{\prime} t^{*} R E_{\text {in }}^{*}\left\{\left|T_{1}\right|^{2}+|t|^{2}\left(\left|T_{2}\right|^{2}-\left|T_{1}\right|^{2}\right)\right\} .
$$

For a 50/50 beam splitter, $|r|^{2}=|t|^{2}=0.5$, Equation (17) reduces to

$$
E_{2}=r^{\prime} t^{*} R E_{\mathrm{in}}^{*}(0.5)\left(\left|T_{1}\right|^{2}+\left|T_{2}\right|^{2}\right),
$$

which is equivalent to addition of the transparency images.

In this configuration, the beam passes through the target twice, once in each direction. For the application of imaging remote targets, the desire is for a setup in which the beam passes through the target only once. This can easily be extended to reflection from a remote target. This configuration also will not remove aberrations from the target. If, for example, the beam passed through a water column, reflected from a target, passed back through the water column and to a detector, an aberrated image would be captured at the detector. If aberrators $\mathrm{T}_{\mathrm{A} 1}$ and $\mathrm{T}_{\mathrm{A} 2}$ are placed in both legs of the interferometer the field $E_{1}$ becomes

$$
E_{1}=|t|^{2} E_{\text {in }}^{*} R r^{*}\left(\left|T_{1}\right|^{2}\left|T_{A 1}\right|^{2}-\left|T_{2}\right|^{2}\left|T_{A 2}\right|^{2}\right) \text {. }
$$

Since $\left|T_{\mathrm{A}}\right|^{2}=1$, by Equation (8), the aberration is removed. In this configuration, there is still a double pass of the beam through the aberrators and the targets.

If the aberrator is placed in front of BS2 the field is

$$
E_{1}=|t|^{2} E_{\text {in }}^{*} R^{*}\left(\left|T_{1}\right|^{2} T_{A}-\left|T_{2}\right|^{2} T_{A}\right)
$$

The beam passes through the aberrator only once, but the aberration is not removed from the collected image. For this configuration, removing aberration from an image can only occur for a double pass of the beam through the aberrator.

\section{IMAGE SUBTRACTION BY SINGLE-PASS DPC}

A method is presented for image subtraction requiring only a single pass through a transmission target with and without an aberrator. A single pass is desired since this is the physical situation in this application. A method for image subtraction with a single pass has been reported ${ }^{15}$; however, phase conjugation requiring two pump beams and a reference beam was used. The DPC technique is desired for ease of alignment. 
Figure 4 shows the experimental setup for image subtraction with a single pass through the targets. The beam is split at BS1 and each resulting beam passes through transparencies $T_{1}$ and $\mathrm{T}_{2}$ only once. $E_{1}$ passes through transparency $\mathrm{T}_{1}$, and $E_{2}$ passes through transparency $\mathrm{T}_{2}$. The beams are recombined at BS2. BS2 has reflection and transmission coefficients $r$ and $t$ for light incident from one side of the cube and $r^{\prime}$ and $t^{\prime}$ for light incident from the other. Lenses L1 and L2 tightly focus the beam into the crystal where the grating is written. $E_{3}$ and $E_{4}$ are the pump beams and also the reference beams. $E_{3}$ is the reference beam for the conjugate of $E_{4}$ while $E_{4}$ is the reference for the conjugate of $E_{3}$ (not used here). The conjugate of $E_{4}$ is passed through BS3 and imaged on the screen S. The distance $l$ is such that $2 l$ is greater than the coherence length of the laser.

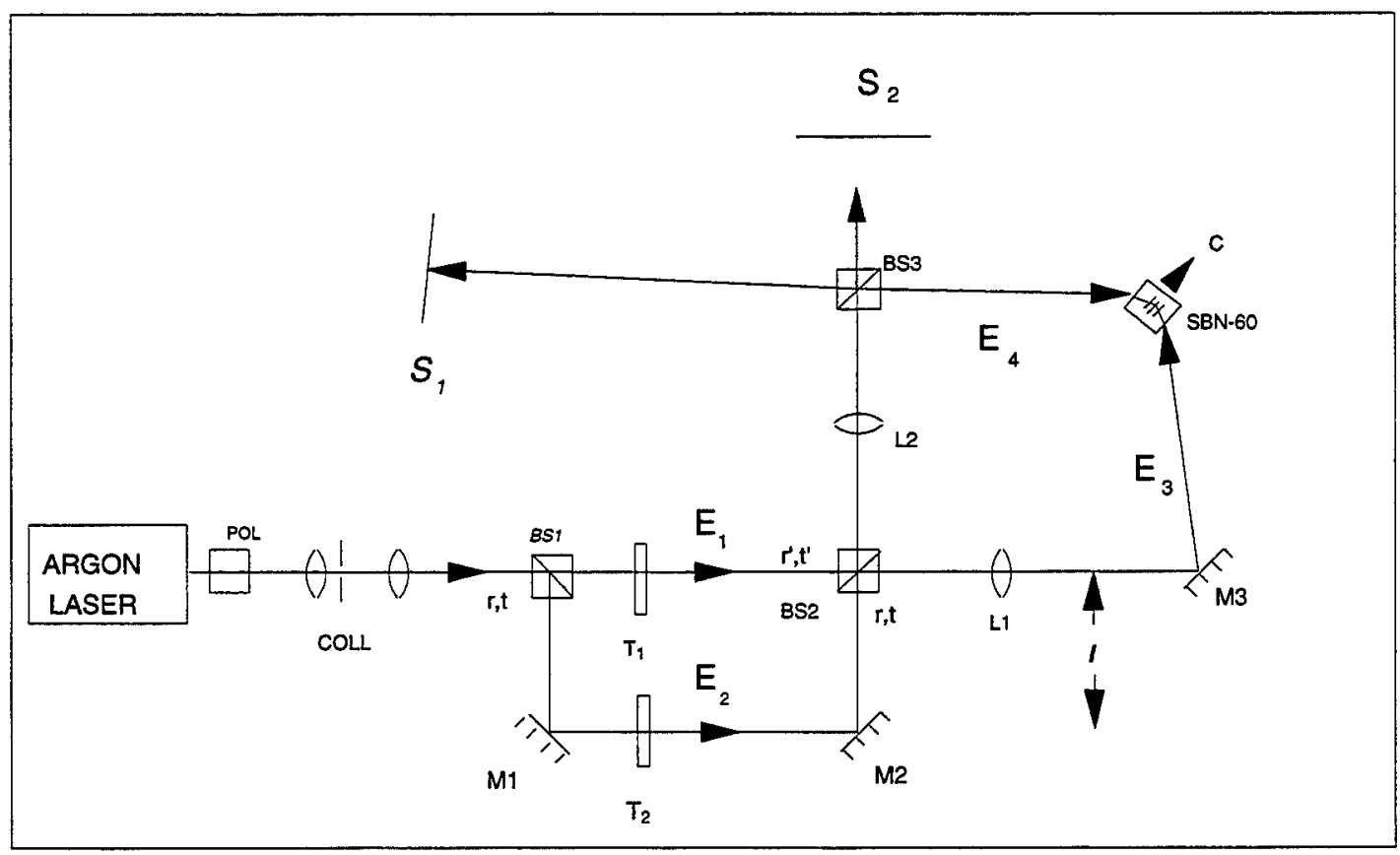

FIGURE 4. EXPERIMENTAL SETUP, SINGLE PASS

If the field incident on $\mathrm{BS} 1$ is denoted $E_{0}$, then the fields $E_{1}$ and $E_{2}$ incident on BS2 are given by

$$
\begin{gathered}
E_{1}=t E_{o} T_{1} \\
E_{2}=r E_{o} T_{2} e^{i k 2 l} .
\end{gathered}
$$

The phase factor in the expression for $E_{2}$ is a consequence of the path difference, $2 l$. The fields, $E_{3}$ and $E_{4}$, incident on the photo refractive crystal are then 


$$
\begin{aligned}
& E_{3}=\left(t^{\prime} E_{1}+r E_{2}\right)=E_{o}\left(t^{\prime} t T_{1}+r^{2} T_{2} e^{i k 2 l}\right) \\
& E_{4}=\left(r^{\prime} E_{1}+t E_{2}\right)=E_{o}\left(r^{\prime} t T_{1}+t r T_{2} e^{i k 2 l}\right)
\end{aligned}
$$

The intensity incident on the crystal is therefore

$$
I=\left|\left(E_{3}+E_{4}\right)\right|^{2} .
$$

The interference between the two fields $E_{3}$ and $E_{4}$ is responsible for writing the grating in the crystal. This is given by the cross terms of Equation (23); i.e.,

$$
I_{\mathrm{int}}=E_{1}^{*} E_{2}+E_{1} E_{2}^{*} \text {. }
$$

This works out to

$$
I_{\text {int }}=I_{o}\left\{\left(t^{\prime \prime \prime} t r^{\prime} t^{\prime \prime}\left|T_{1}\right|^{2}+|r|^{2} r^{\prime \prime} t\left|T_{2}\right|^{2}\right)+c . c .\right\}
$$

where the mutually incoherent cross terms have been neglected. For DPC, it is not necessary for the pump beams to be mutually incoherent, as in standard four-wave mixing; however, it is necessary here to neglect these cross terms. This is accomplished by ensuring the path difference $2 l$, is greater than the coherence length of the laser.

Applying Stoke's principle of time reversibility of light from Equation (11) to the expression for the intensity in Equation (25)

$$
I_{\text {int }}=I_{o}\left\{r^{*} t\left(|r|^{2}\left|T_{1}\right|^{2}-|t|^{2}\left|T_{2}\right|^{2}\right)+c . c .\right\} .
$$

The negative sign in Equation (26) indicates a phase difference of $\pi$ between the two beams carrying the image information from transparencies $T_{1}$ and $T_{2}$. This implies that the holographic grating containing the image of $T_{1}$ is shifted 180 deg relative to the holographic grating containing the image of $T_{2}$. When the holograms are read out by the reference beam (one of the pump beams in this case), the result is the difference between the two intensity patterns provided $|r|^{2}=|t|^{2}(50 / 50$ beam splitters $)$.

The subtraction of the field amplitudes can be achieved by inserting a spatial filter in one arm of the interferometer. Inserting a pinhole at the focus of L1 destroys the spatial information for $E_{3}$ so that

$$
E_{4}=E_{o}\left(r^{\prime} t+t r e^{i k 2 l}\right)
$$


Inserting Equation (27) into Equation (23) and assuming the beam splitters are 50/50,

$$
I_{\text {int }}=I_{o}\left\{r^{*} t|t|^{2}\left(T_{2}-T_{1}\right)+\text { c.c. }\right\}
$$

for the interference intensity.

As in the previous configuration, removing target $T_{1}$ results in the inversion of image $T_{2}$. Removing both targets results in total destructive interference of the two Gaussian beams. This is demonstrated by the data in Figures 5a-f. Referring back to Figure 3, assign $E_{1}$ to beam $A$ and $E_{2}$ to beam $B$. No targets or aberrators are present. Figures $5 \mathrm{a}$ and $\mathrm{c}$ are the Gaussian beams $\mathrm{A}$ and $B$. The image of beam A was taken by placing a charge couple device (CCD) camera at the output S2 from BS3 in Figure 3 while blocking beam B. The output of the CCD was input to a video screen and a photograph was taken of the screen. The image of beam $B$ was taken in the same manner while blocking beam $A$. The conjugate images were taken at the other output of BS3 at S1 and are shown in Figures $5 b$ and d. Figure $5 e$ is the superposition of beams A and B taken at $\mathrm{S} 2$ without blocking any beams. Figure $5 \mathrm{f}$ is the conjugate of $\mathrm{A}+\mathrm{B}$ showing the subtraction of the two Gaussian beams.

It has been demonstrated that image subtraction by DPC with a single pass through the targets is possible with DPC. Attention is turned to verifying whether this method works to remove aberrations from an image. If aberrators $\mathrm{T}_{\mathrm{A} 1}$ and $\mathrm{T}_{\mathrm{A} 2}$ are placed in the paths of $E_{1}$ and $E_{2}$, Equation (26) becomes

$$
I_{\text {int }}=I_{o}\left\{r^{*} t\left(\left|r T_{2} T_{A 2}\right|^{2}-\left|t T_{1} T_{A 1}\right|^{2}\right)+c . c .\right\} .
$$

This single pass configuration does remove the aberrations. Notice that if only one leg of the interferometer has a target and aberrator, the aberration is still removed and the image is inverted. If the pinhole is used along with the aberrators, Equation (29) becomes

$$
I_{\text {int }}=I_{o}\left\{r^{*} t^{2}\left(|r|^{2} T_{2} T_{A 2}-|t|^{2} T_{1} T_{A 2}\right)+c . c .\right\} .
$$

The pinhole configuration does not eliminate the aberration. Because of time constraints, this result was unable to be experimentally verified.

\section{CONCLUSIONS}

Image subtraction has been demonstrated for two configurations. In one configuration, the beam was transmitted through the target twice and in the other only once. It has also been shown that, for the single-pass configuration, an aberration can be removed from an image. This can be applied to the problem of remote imaging by having one leg of the interferometer in Figure 4 be the return signal + noise from a remote target. The second leg is split from the beam 


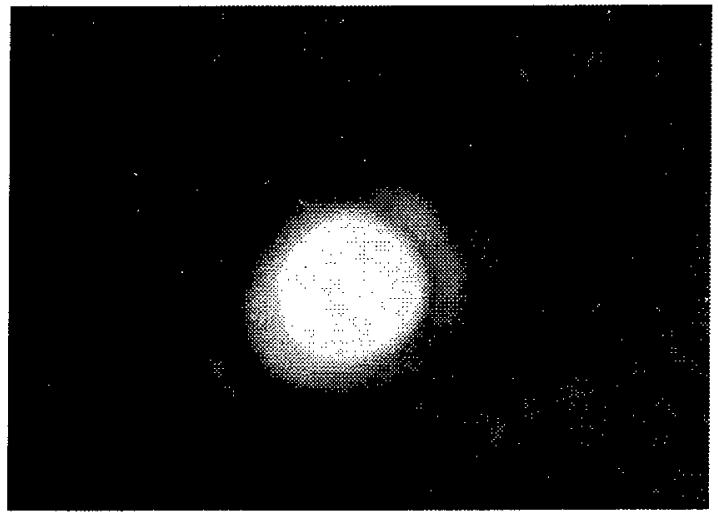

(a) BEAM A

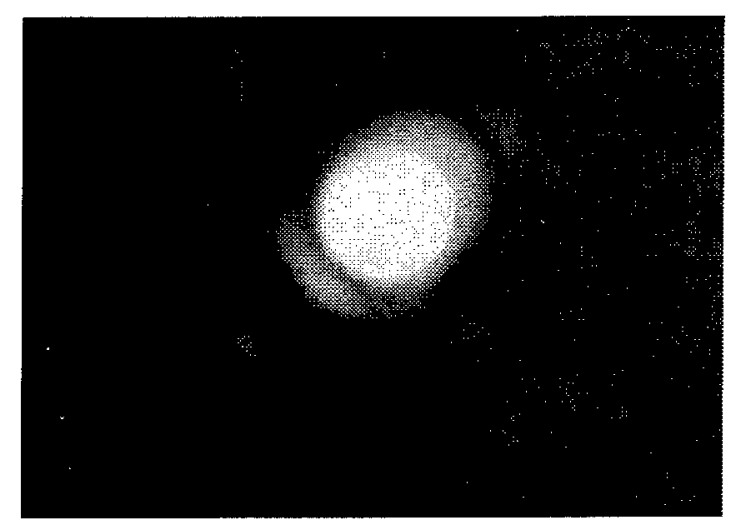

(c) BEAM B

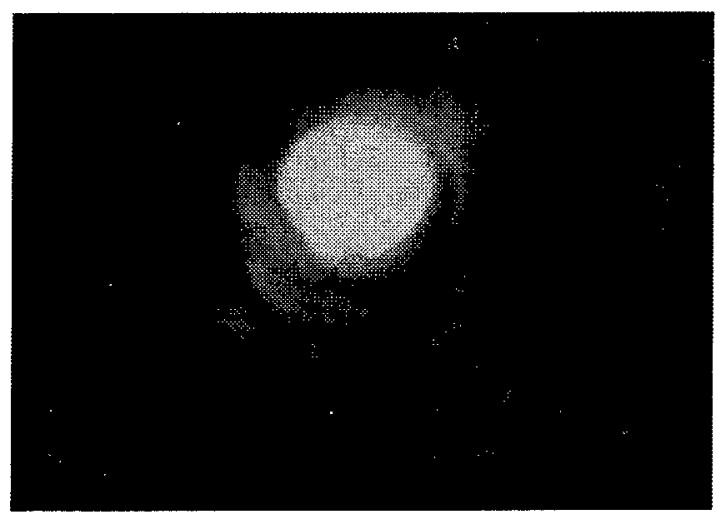

(e) BEAMS A + B

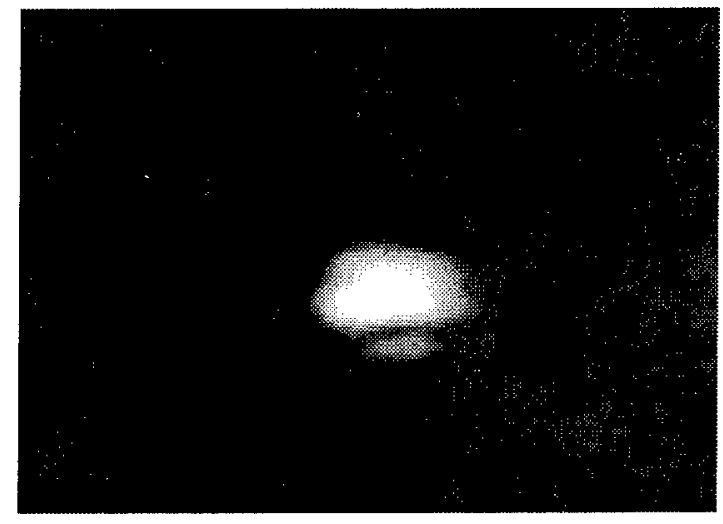

(b) CONJUGATE OF BEAM A

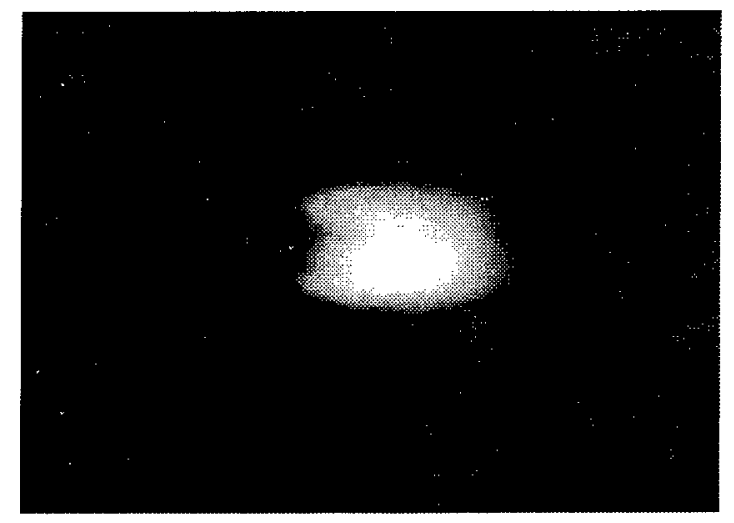

(d) CONJUGATE OF BEAM B

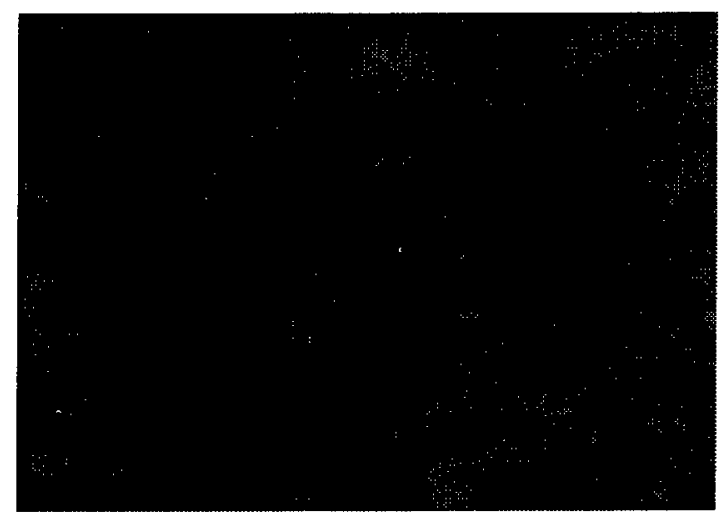

(f) CONJUGATE OF BEAMS A + B

FIGURE 5. SUBTRACTION OF TWO GAUSSIAN BEAMS 
before sending it to the target. The path difference is much greater than the coherence length of the laser. The two beams are recombined at BS2 and focussed on the conjugate mirror. The output at $S_{1}$ will be the image of the target with the aberration removed. There will be some slight attenuation from absorption that has not been included in these calculations.

The condition (Equation (8)) that the type of aberration must be a phase distortion is stressed. Spatially varying amplitude aberrations will not be removed or reduced. Aberrations induced by a beam traversing a water column are of both types. More data is needed to determine whether this is a viable technique for underwater imaging.

The response of this device is dependent on the time it takes for the grating to be written in the crystal. This is proportional to the intensity of the writing beams. With the argon-ion laser operating at $80 \mathrm{~mW}$, this time was on the order of several minutes. Since the return from a target will have a much lower intensity than a laser in the laboratory, this is an unacceptable condition. It is believed that this configuration has promise for image enhancement applications if the response time can be improved. The response is also a function of the type of doubling crystal used. Other non-linear materials have faster response times. 


\section{REFERENCES}

1. Fisher, R. A., ed., Optical Phase Conjugation, Academic Press, New York, 1983, Chapter 1.

2. Weiss, S.; Sternklar, S.; and Fisher, B., Opt. Lett. 12, 114, 1987.

3. Smout, A. M. C. and Eason, R. W., Opt. Lett. 12, 498, 1987.

4. Shkunov, V. V. and Zel'dovich, B. Y., Sci. Amer. 54, Dec 1985.

5. $\quad$ Pepper, D. M., Sci. Amer. 74, Jan 1986.

6. Feinberg, J., Physics Today, 46, Oct 1988.

7. Sharp, E. J.; Miller, M. J.; Salamo, G. J.; Clark III, W. W.; and Wood, G. L., Ferroelectrics, 87, 335, 1988.

8. Weiss, S.; Werner, O.; and Fischer, B., Appl. Phys. Lett. 50, 483, 1987.

9. Hamasaki, J., Appl. Opt. 7, 1613, 1968.

10. Sternklar, S.; Weiss, S.; and Fischer, B., Opt. Eng. 26, 423, 1987.

11. Weiss, S.; Sternklar, S.; and Fischer, B., Opt. Lett. 12, 114, 1987.

12. Kramer, M.; Alley, T. G.; Martinez, D. R.; and Schelonka, L. P., Appl. Opt. 29, 2576, 1990.

13. Kwong, S. K.; Rakujic, G. A.; and Yariv, A., Appl. Phys. Lett., 48, 201, 1986.

14. Sharp, E. J.; Clark III, W. W.; Miller, M. J.; Wood, G. L.; Monson, B.; Salamo, G. J; and Neurgaonkar, R. R., Appl. Opt., 29, 743, 1990.

15. Yeh, P.; Chang, T.Y.; and Beckwith, P.H., Opt. Lett., 13, 586, 1988. 


\section{DISTRIBUTION}

\section{$\underline{\text { Copies }}$}

DOD ACTIVITIES (CONUS)

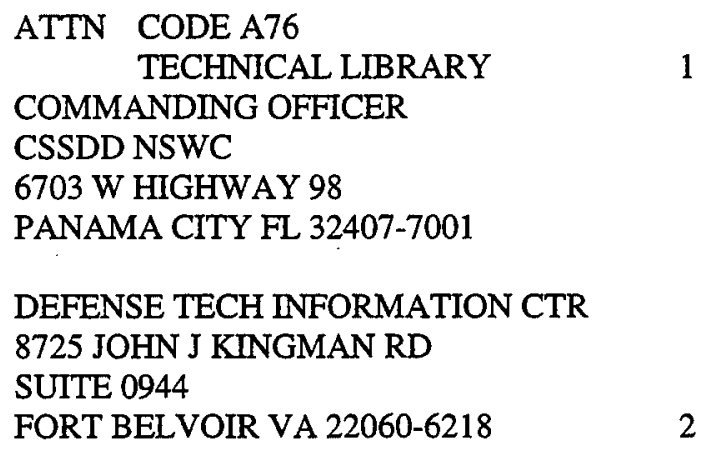

NON-DOD ACTIVITIES (CONUS)

THE CNA CORPORATION

P O BOX 16268

ALEXANDRIA VA 22302-0268

ATTN ME STEFANOV

KENT DISPLAY SYSTEMS

343 PORTAGE BLVD

BRIMFIELD OH 44240

\section{INTERNAL}

B20 (SWANSON)

2

B60 (TECHNICAL LIBRARY) 3 